\title{
Predictores de requerimiento intraoperatorio de soporte inotrópico y/o vasopresor en cirugía de revascularización miocárdica
}

\author{
Predictors for intraoperative requirement of inotropic and / or vasopresor \\ support in miocadical revascularization surgery
}

Fabio A. Sepúlveda L. MD. ${ }^{1, *}$, Luz M. Jiménez M. MD. ${ }^{1}$, Hernán D. Castro A. MD. ${ }^{2}$, Jorge A. Castro P. MD. ${ }^{3}$, Eliana M. Cañas A. Mg. ${ }^{3}$, Junior E. Hidalgo O. MD. ${ }^{3}$, Manuela Guzmán C. ${ }^{4}$, Jesús S. Gómez Z. ${ }^{4}$

Universidad Pontificia Bolivariana. Medellín, Colombia.

Clínica Medellín QuironSalud, Universidad CES. Medellín, Colombia.

Clínica CardioVID. Medellín, Colombia.

4 Facultad de Medicina, Universidad Pontificia Bolivariana.

Fuente de financiamiento: Este trabajo no tuvo ninguna fuente de financiamiento.

Declaración de conflicto de intereses: Los autores declaran no tener ningún conflicto de intereses.

Fecha de recepción: 01 de marzo de 2021 / Fecha de aceptación: 10 de mayo de 2021

\begin{abstract}
Objective: To determine predictive factors associated with the need for inotropic and/or vasopressor intraoperative support in patients undergoing myocardial revascularization surgery. Methods: Retrospective cohort analytical study with nested analysis of cases and controls of patients older than 18 years scheduled for elective myocardial revascularization surgery between 2014 and 2017. The incidence of inotropic and/or vasopressor intraoperatively was established and bivariate analysis for possible predictors was performed. Results: 466 patients were studied. The requirement of inotropics and / or vasopressors during the intraoperative period was 75\% (350 patients). The use of beta blockers increases the risk 1.85 times of requiring inotropics and / or vasopressors ( $p$ 0.01). Conclusions: Chronic use of beta blockers before myocardial revascularization with extraocorporeal circulation increases the need for the use of inotropics and / or vasopressors. Other factors described in the literature did not have statistical significance in this study.
\end{abstract}

Key words: Perioperative period, coronary heart disease, hypotension, metoprolol.

\section{RESUMEN}

Objetivo: Determinar factores predictores asociados a la necesidad de soporte inotrópico y/o vasopresor intraoperatorio en pacientes sometidos a cirugía de revascularización miocárdica. Métodos: Estudio analítico de cohorte retrospectiva con análisis anidado de casos y controles de pacientes mayores de 18 años programados para cirugía electiva de revascularización miocárdica entre los años 2014 y 2017. Se estableció la incidencia del uso de inotrópicos y/o vasopresores en el intraoperatorio y se realizó análisis bivariado para posibles predictores. Resultados: Se estudiaron 466 pacientes. El requerimiento de inotrópicos y/o vasopresores durante el intraoperatorio fue $75 \%$ (350 pacientes). En este estudio se encontró que el uso de betabloqueadores crónicamente aumento el riesgo en 1,85 veces de requerir inotrópicos y/o vasopresores ( $\mathrm{p} 0,01)$. Conclusiones: El uso crónico de betabloqueadores antes de la revascularización miocárdica con circulación extracorpórea aumenta la necesidad de la utilización de inotrópicos y/o vasopresores. Otros factores predictores descritos en la literatura no tuvieron en este estudio significancia estadística.

Palabras clave: Período perioperatorio, enfermedad coronaria, hipotensión, metoprolol. 


\section{Introducción}

a enfermedad coronaria sigue siendo una de las primeras causas de muerte en el mundo, sin embargo, los múltiples tratamientos disponibles en la actualidad han reducido la morbimortalidad en la población que la padece[1]. La cirugía de revascularización miocárdica con circulación extracorpórea (CEC) es una de las estrategias terapéuticas para el manejo de esta enfermedad y sus resultados en términos de morbimortalidad a nivel mundial se reporta por debajo del 4\% según la Sociedad Americana de Cirujanos de Toráx (STS)[2].

En un reporte realizado por Menéndez, et al., relacionado con el registro de cirugías cardiovasculares realizadas en el 2016 en España, se reportó que, en cirugía cardiaca mayor, la tasa de mortalidad fue del 5,6\%[3].

Las tasas reportadas anteriormente, van de la mano con el uso de inotrópicos y/o vasopresores (IYOV) durante el intraoperatorio dado que al reducir el riesgo de complicaciones perioperatorias se mejoran las condiciones clínicas del paciente cuando éste presente alteraciones hemodinámicas que comprometan la función cardiaca[4],[5]. Con los fármacos vasopresores se busca vasoconstricción periférica, incremento de la resistencia vascular sistémica y la presión arterial media; con los inotrópicos se busca un incremento en la contractilidad cardíaca y la presión arterial media. Estos efectos constituyen la piedra angular en la preservación de órganos vitales manteniendo su adecuada perfusión[6]. Sin embargo, también es importante mencionar que este tipo de fármacos no están ajenos a generar complicaciones, pues además de aumentar el riesgo de arritmias, producen cambios fisiológicos en el metabolismo de los carbohidratos, en el funcionamiento de la glándula tiroides, del sistema inmunológico y de la coagulación[7].

En los últimos años se han venido realizando diferentes estudios que describen factores de riesgo asociados al uso de IYOV (inotrópicos y/o vasopresores) en el perioperatorio de cirugía cardiovascular. La identificación de estos predictores puede llevar a establecer estrategias de manejo que disminuyan la tasa de complicaciones.

El estudio de Ahmed et al., reportó las siguientes condiciones hemodinámicas y clínicas como predictores del uso de IYOV en el intra o postoperatorio inmediato: el índice cardiaco $\leq 2,5$ $\mathrm{L} / \mathrm{min} / \mathrm{m}^{2}$, la presión diastólica final del ventrículo izquierdo $\geq 20$ $\mathrm{mm} \mathrm{Hg}$, fracción de eyección del ventrículo izquierdo $\leq 40 \%$ y enfermedad renal crónica en estadio mayor de 3[8].

Estudios como el realizado por Alborz et al., han descrito que los betabloqueadores con propiedades cronotrópicas negativas generan menor demanda de oxígeno por el miocardio, tienen efectos antiarrítmicos, antiinflamatorios y por ende, en cuanto a la reducción de la mortalidad en pacientes de alto riesgo, pueden comportarse como un factor protector, no obstante su uso en el intraoperatorio aún genera preocupación por el riesgo de hipotensión intraoperatoria y bradicardia[9].

El objetivo del presente estudio es establecer predictores preoperatorios asociados al requerimiento intraoperatorio de IYOV en pacientes llevados a cirugía de revascularización miocárdica en un centro de referencia de la ciudad de Medellín. Se analizan las características demográficas, la frecuencia del requerimiento de IYOV, el tipo de fármaco usado y las complicaciones asociadas a su uso.

\section{Metodología}

\section{Diseño y población del estudio}

Se realizó un estudio analítico de cohorte retrospectiva con análisis anidado de casos y controles; definiendo como caso los pacientes que desde el momento de la cirugía de revascularización coronaria y hasta el posoperatorio inmediato recibieron tratamiento farmacológico de tipo inotrópico y/o vasopresor, definido como el requerimiento en infusión continua de uno de los siguientes fármacos: dobutamina, milrinone, levosimendan, dopamina, epinefrina y norepinefrina; y definiendo como control los pacientes que desde el momento de la cirugía y hasta el posoperatorio inmediato no requirieron la administración de tratamiento farmacológico de tipo inotrópico o vasopresor.

Fueron incluidos pacientes mayores de 18 años programados de forma electiva para cirugía de revascularización miocárdica en el período comprendido entre enero de 2014 y enero de 2017 en un centro de referencia de la ciudad de Medellín. Se excluyeron aquellos pacientes llevados a cirugía de forma emergente, a quienes se les realizó cirugía valvular concomitante, los que estaban recibiendo soporte IYOV previamente, o aquellos que tenían balón de contrapulsación intraaórtica.

\section{Proceso de recolección}

Una vez se obtuvo el aval de ética por la institución participante y se tuvo acceso a las historias clínicas del servicio de cardiología, se verificaron los criterios de inclusión y exclusión. La información recolectada se registró en Microsoft ${ }^{\circledR}$ Excel clasificada según las variables, donde se comprobó la calidad de la información.

Las variables de interés evaluadas fueron: Características demográficas (edad, sexo, peso, talla, índice de masa corporal antes de cirugía, clase funcional de la NYHA y clasificación ASA antes de cirugía). Parámetros de laboratorio (hemoglobina preoperatoria, tasa de filtración glomerular preoperatoria calculada por la ecuación CKD EPI, hemoglobina glicosilada preoperatoria). Resultados de ecocardiografía transtorácica preoperatoria (fracción de eyección del ventrículo izquierdo - FEVI, presencia o no de trastornos segmentarios de contractilidad, presencia o no de insuficiencia mitral caracterizada como leve, moderada o severa, presencia o no de hipertensión pulmonar probable determinada por estimación de presión sistólica de la arteria pulmonar mayor de $40 \mathrm{mmHg}$. Tiempo de circulación extracorpórea, tiempo de pinzamiento aórtico, tiempo de estancia en UCl (unidad de cuidado intensivo) luego de cirugía, tiempo de estancia hospitalaria total posoperatoria y el soporte hemodinámico farmacológico, definiendo el uso o no de uno o más de los siguientes fármacos: dobutamina, milrinone, levosimendan, dopamina, epinefrina, norepinefrina.

\section{Análisis estadístico}

Las variables cualitativas se expresaron mediante frecuencias absolutas y relativas; las cuantitativas por medio de mediana y los percentiles 25 y 75 teniendo en cuenta que los datos no tuvieron distribución normal (prueba estadística de Kolmogorov-Smirnov $p<0,05$ ).

Se establecío si había asociación entre los diferentes factores analizados y el uso o no de inotrópicos y/o vasopresores utilizando la prueba de Chi cuadrado, se calcularon los Odds Ratio (OR) con sus respectivos intervalos de confianza al 95\%. 
Se estableció como nivel de significancia estadística p menor de 0,05. El análisis estadístico se realizó mediante el software Statistical Package for the Social Science SPSS versión 22.

El presente estudio fue aprobado por el Comité de Ética de Investigación en Salud de la Universidad Pontificia Bolivariana y se obtuvo el aval de la institución participante.

\section{Resultados}

Entre el periodo del estudio, fueron intervenidos quirúrgicamente 698 pacientes de los cuales 466 cumplieron con los criterios de elegibilidad. El proceso de selección de la población de estudio se describe en la Figura 1.

\section{Características sociodemográficas y clínicas}

El 43\% (200 pacientes) eran mayores de 65 años, 355 (76\%) de los pacientes eran de sexo masculino, 353 (86\%) pacientes tenían como antecedente personal hipertensión arterial, los demás datos demográficos y clínicos se presentan en la Tabla 1. Del total de pacientes incluidos, 311 de ellos $(66,7 \%)$ se encontraron en una clase funcional II de la NYHA (New York Heart Association) y 389 (83,5\%) pacientes tenían un estado funcional III según la ASA (American Society of Anesthesiologist).

\section{Condiciones preoperatorias}

Se reportaron trastornos segmentarios de la contractilidad en 273 pacientes $(58,6 \%)$, de los cuales, $208(59,4 \%)$ requi-

\begin{tabular}{lc}
\multicolumn{2}{c}{ Tabla 1. Características sociodemográficas y clínicas } \\
\hline Variable & $\mathbf{n}(\%)$ \\
Sociodemográficas & $64(57-71)$ \\
Edad & $72(64-80)$ \\
Peso & $355(75,1)$ \\
Sexo masculino & $1,66(1,66-1,71)^{*}$ \\
Talla & $26(24,17-28,70)^{*}$ \\
IMC & \\
Clínica & $104(22,3)$ \\
Tabaquismo activo & $353(8,6)$ \\
Hipertensión & $150(32,2)$ \\
Diabetes mellitus & $80(17,2)$ \\
Insulinorrequiriente & $95(41,8)$ \\
Uso de BB preoperatorio &
\end{tabular}

*Mediana (RIQ); BB: betabloqueadores; IMC: Índice de masa corporal.

rieron soporte IYOV en el intraoperatorio. De 100 pacientes documentados con valvulopatía mitral, 86 de ellos tenían insuficiencia mitral leve y 70 requirieron soporte IYOV. Cuarenta y dos pacientes tenían fracción de eyección reducida o inferior al 40\%, 35 de ellos (83,3\%), requirieron soporte IYOV. Las dem\{as condiciones preoperatorias se presentan en la Tabla 2.

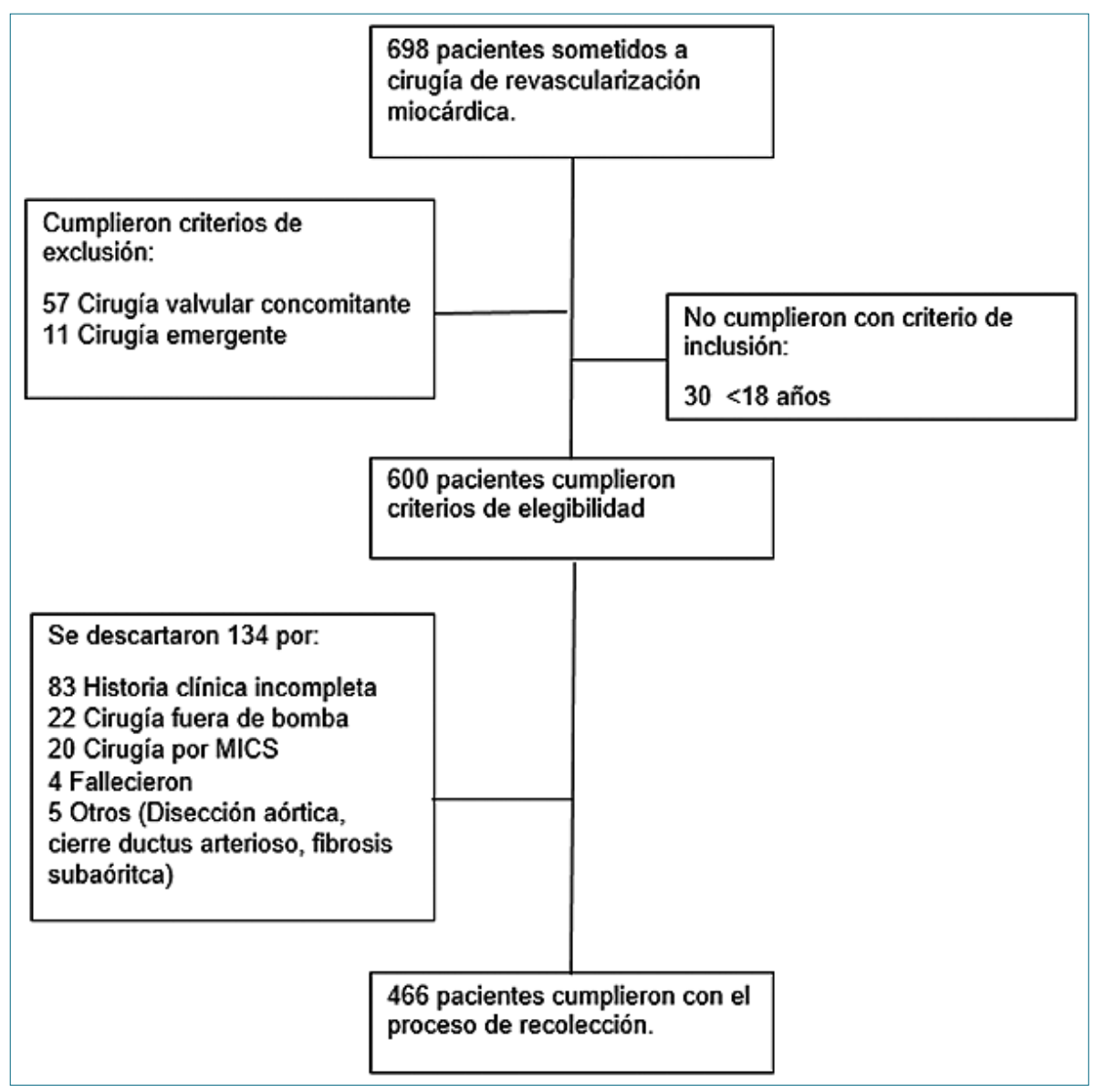

Figura 1. Proceso de selección de los pacientes. 


\begin{tabular}{lc}
\multicolumn{2}{c}{ Tabla 2. Condiciones preoperatorias } \\
\hline Variable & $\mathbf{n}(\%)$ \\
Laboratorios & \\
$\mathrm{Hb}(\mathrm{mg} / \mathrm{dl})$ & $14,3(13,1-15,42) *$ \\
$\mathrm{TFG}$ & $84(70-95,2) *$ \\
$\mathrm{HbA} 1 \mathrm{C}$ & $5,9(5,6-6,5)$ * \\
Ecográficas & \\
FEVI (\%) & $0,55(0,45-0,60)$ * \\
Trastornos segmentarios & $273(58,6)$ \\
Insuficiencia mitral & \\
Leve & $86(18,5)$ \\
Moderada & $11(2,4)$ \\
Severa & $3(0,60)$ \\
Hipertensión pulmonar & $40(8,6)$ \\
\hline
\end{tabular}

*Mediana (RIQ); Hb: hemoglobina; FEVI: Fracción de eyección ventricular izquierda; $\mathrm{HbA} 1 \mathrm{C}$ : hemoglobina glicosilada.

\begin{tabular}{lc}
\multicolumn{2}{c}{ Tabla 3. Soporte hemodinámico y variables de tiempo } \\
intraoperatorio \\
\hline Variables & $\mathbf{n}(\%)$ \\
Soporte IYOV & $5(1,10)$ \\
Dopamina & $196(42,1)$ \\
Dobutamina & $15(3,20)$ \\
Milrinone & $15(3,20)$ \\
Levosimendan & $307(65,90)$ \\
Norepinefrina & $3(0,60)$ \\
Adrenalina & \\
Tiempo & $81,5(69-100)^{*}$ \\
CEC (minutos) & $66(53-85)^{*}$ \\
Pinzamiento aórtico & $2(1-3)$ \\
Estancia en UCI POP (días) & $6(4-9)$ \\
Estancia hospitalaria &
\end{tabular}

*Mediana (IQR); CEC: circulación extracorpórea; POP: posoperatorio; UCI: Unidad de Cuidado Intensivo.

\begin{tabular}{lcc}
\hline & Tabla 4. Riesgo de requerir inotrópico y/o vasopresor intraoperatorio \\
\hline Variable & OR (IC 95\%) & Valor $\mathbf{p}$ \\
Uso de BB preoperatorios & $1,85(1,184-2,89)$ & 0,006 \\
CEC menor de 60 min & $2,149(1,061-4,355)$ & 0,030 \\
CEC entre 60 y 120 & $0,463(0,269-0,797)$ & 0,005 \\
Pinzamiento menor de 60 min & $1,673(1,047-2,674)$ & 0,03 \\
Pinzamiento entre 60 y 120 min & $0,541(0,34-0,859)$ & 0,009 \\
Mayor de 70 años & $1,484(0,906-2,428)$ & 0,123 \\
Trastornos segmentarios & $1,149(0,752-0,757)$ & 0,52 \\
FEVI menor de 40 & $1,73(0,747-4,009)$ & 0,261 \\
\hline
\end{tabular}

CEC: circulación extracorpórea; prueba de Chi cuadrado.

\section{Variables de tiempo intraoperatorio.}

El tiempo promedio de CEC fue de 81,5 minutos (69-100) y el de pinzamiento aórtico de 66 minutos (53-85). De estos pacientes aquellos con CEC menor de 120 minutos y tiempo de pinzamiento aórtico menor de 120 minutos tuvieron menos requerimiento de IYOV. OR 0,46, IC 0,26-0,79 ( $p=0,005)$ y OR $0,54, I C 0,34-0,85$ ( p 0,009) respectivamente (Tablas 3 y 4).

\section{Soporte hemodinámico}

La frecuencia de requerimiento de soporte IYOV durante el intraoperatorio fue del 75\%. El fármaco vasopresor más usado fue norepinefrina en 307 pacientes (66\%), mientras que el inotrópico de mayor uso fue dobutamina en 192 pacientes (42\%) como se evidencia en la Tabla 3.

\section{Complicaciones posoperatorias}

Dentro de las $72 \mathrm{~h}$ posteriores a la intervención quirúrgica, 82 pacientes $(17,6 \%)$ presentaron algún tipo de complicación. La complicación más frecuente fue la presencia de fibrilación auricular en 47 pacientes (10\%) de los cuales 41 había requerido soporte IYOV en el intraoperatorio. Otras complicaciones fueron mucho menos frecuentes como infarto perioperatorio
11 pacientes $(2,4 \%)$, bradiarritmias 3 pacientes $(0,6 \%)$, taquicardia ventricular 2 pacientes $(0,4 \%)$ entre otras con menor presentación.

\section{Riesgo de requerir inotrópico y/o vasopresor en el intraoperatorio}

El riesgo de requerir soporte IYOV en nuestro medio esta condicionado por múltiples factores, sin embargo, encontramos que este riesgo se incrementa en los pacientes que reciben betabloqueadores antes de la cirugía comparado con los que no los vienen recibiendo de forma crónica. En cuanto a las otras variables preoperatorias analizadas que eran el objetivo de nuestro estudio, no se encontró significancia estadística. (Tabla 4).

\section{Discusión}

En cuanto al tiempo de CEC y tiempo de pinzamiento aórtico (TPA), en estudios previos no se encontró relación entre estos tiempos y el uso de IYOV[8] u otros riesgos de complicaciones posoperatorias tales como septicemia, complicaciones 
renales, accidentes cerebrovasculares o muerte[10]. El presente estudio pudo establecer que un tiempo de pinzamiento aórtico inferior a 120 minutos se comporta como factor protector que disminuyen el riesgo de uso de soporte IYOV.

Si bien es cierto, la cirugía cardiaca con CEC puede desencadenar un síndrome de respuesta inflamatoria sistémica con disfunción multiorgánica e hipotensión grave[11], la manipulación cardiaca realizada en los pacientes fuera de CEC, conduce a un deterioro hemodinámico significativo que es aún más pronunciado cuando se requiere una manipulación extensa del corazón[12]. Cuando los pacientes están en CEC tienen menos probabilidad de presentar coagulopatía caracterizada por coagulación microvascular, disfunción plaquetaria y fibrinólisis aumentada, la cual es controlada mediante la administración de heparina durante el procedimiento[10], lo anterior puede explicar en parte porqué en este estudio, el tiempo de CEC menor de 120 minutos se comportó como factor protector que disminuye el riesgo de requerir IYOV al igual que el TPA.

En el presente estudio se evidenció que el $45,4 \%$ de los pacientes que usaban betabloqueadores en el preoperatorio requirieron soporte IYOV. En términos de riesgo, el uso preoperatorio de este grupo de medicamentos aumenta 1,85 veces más la probabilidad de requerir soporte IYOV $(p<0,05)$ que quienes no los reciben previamente, riesgo que se correlaciona con lo reportado en la literatura.

Hay trabajos publicados en los cuales se menciona que la vasoplejia es una complicación asociada al uso de betabloqueadores en el preoperatorio de cirugía cardiovascular[13],[14]. Alborz et al.[8], aclara que aunque los beta bloqueadores por sus propiedades cronotrópicas negativas generan menor demanda de oxígeno y tienen efectos antiarrítmicos y antiinflamatorios, en cirugía no cardiaca persiste la preocupación por el aumento del riesgo de hipotensión intraoperatoria y bradicardia que requieran el uso de IYOV para tratar estas complicaciones. De igual manera, el estudio POISE[15] reportó que en cirugía no cardiaca, el uso de metoprolol reducía el riesgo de infarto agudo de miocardio pero también se observó incremento en la mortalidad y accidentes cerebrovasculares relacionados con hipotensión.

La American College of Cardiology (ACC) y la American Heart Association (AHA)[16], reportaron que en cirugía no cardíaca, el uso de betabloqueadores incrementa el riesgo de hipotensión y el riesgo de bradicardia.

En el metaanálisis desarrollado por Blessberger, et al[17] EMBASE, the Cochrane Central Register of Controlled Trials (CENTRAL, se evaluó el efecto de los betabloqueadores en el perioperatorio tanto en cirugía cardiaca como en intervenciones no cardiacas y no se logró establecer un papel definitivo de los beta bloqueadores en mortalidad, prevención de IAM, causar accidentes cerebrovasculares, hipotensión y bradicardia. Dentro de su análisis plantean que es posible que el traumatismo quirúrgico, en lugar de los beta bloqueadores, sean un cofactor importante para estos eventos. Sin embargo, en el presente estudio se logró demostrar con significancia estadística, que el uso de betabloqueadores preoperatorios aumentan el riesgo de requerir soporte vasopresor y/o inotrópico en el intraoperatorio.

Aunque se ha descrito que luego de cirugía cardiovascular las complicaciones son atribuidas a cambios en la precarga, poscarga, frecuencia cardíaca y el estado inotrópico o lusitrópico del corazón[14], es claro que además del fenómeno infla- matorio que genera la cirugía de revascularización coronaria con CEC[18], los fármacos vasopresores e inotrópicos afectan el funcionamiento del sistema de conducción aumentando el riesgo de fibrilación auricular en estos escenarios. En nuestro estudio la presencia de fibrilación auricular se encontró en 47 pacientes, de los cuales 41 habían requerido soporte IYOV en el intraoperatorio. Esto podría representar un riesgo a mediano plazo para esta población, ya que algunos estudios han reportado que dentro de los predictores de mortalidad posterior al procedimiento quirúrgico (hasta un año después del mismo), el antecedente de fibrilación auricular aumentó el riesgo de muerte en 1,5 veces[19].

Con respecto a la función ventricular disminuida, el estudio publicado por Ahmed et al,[8], pudo demostrar que el tener una fracción de eyección del ventrículo izquierdo (FEVI) inferior al $40 \%$ se comporta como predictor independiente del uso de inotrópicos luego de bypass coronario. En el presente estudio, de 466 pacientes analizados, solo 42 tenían FEVI inferior al $40 \%$ y de este subgrupo, el $83,3 \%$ requirió soporte IYOV. Los 424 restantes tenían FEVI superior al 40\%. Lo anterior puede explicar en parte, el porqué este trabajo no logró establecer con significancia estadística que el tener una FEVI reducida o inferior al $40 \%$ se comporte como predictor del uso de IYOV, quizás por el reducido tamaño de la muestra. Sin embargo, este dato es importante, porque si se establecen estrategias eficaces de manejo para optimizar la FEVI preoperatoria en el paciente con enfermedad coronaria, el pronóstico sería más favorable.

Las limitaciones que tiene este trabajo es que los datos se recogieron de una sola institución.

Si bien es cierto que usar betabloqueadores en el preoperatorio arrojó resultados estadísticamente significativos, otra debilidad encontrada es que no se describen las dosis, si existía diferencia entre el betabloqueador recibido (bisoprolol, metoprolol u otro), el tiempo de uso del medicamento y el tiempo de suspensión antes de la cirugía.

Lo más frecuente en nuestro sistema de salud en Colombia es que el paciente reciba metoprolol, el cual es metabolizado en el hígado por el citocromo P450 2D6 (CYP2D6) cuya molécula está sujeta a polimorfismo genético y en individuos identificados como pobres metabolizadores se aumentan los niveles de este medicamento en plasma. La manifestación clínica de esta variabilidad genética se traduce en una baja significativa de la frecuencia cardiaca, presión arterial diastólica y aumento de arritmias en los pacientes con infartos recientes[20].

Tampoco se describen las dosis de soporte IYOV requeridas y las indicaciones hemodinámicas por las cuales el anestesiólogo decidió iniciarlo.

No obstante, a pesar de las debilidades descritas anteriormente, los resultados mostrados en este trabajo se convierten en una herramienta de gran valor para el médico especialista en cuidado perioperatorio quien en última instancia valora los múltiples riesgos que existen alrededor de un paciente con enfermedad coronaria que va para cirugía de revascularización y lo fortalece en la adecuada toma de decisiones en la valoración preoperatoria.

\section{Conclusión}

Los pacientes con enfermedad coronaria que son candida- 
tos a cirugía de revascularización miocárdica con CEC y que toman medicamentos betabloqueadores antes del procedimiento quirúrgico, tienen riesgo aumentado de requerir soporte vasopresor y/o inotrópico en el intraoperatorio.

Agradecimientos: A la Clínica CardioVID por su colaboración en la captación de pacientes.

\section{Referencias}

1. Colombia. Ministerio de Salud y Protección Social. Carga de la enfermedad por enfermedad crónica no transmisible y discapacidad en Colombia. Bogotá: ONS; 2015.

2. A. Laurie W. Shroyer, Laura P. Coombs, Eric D. Peterson, et al. The society of thoracic surgeons: 30-day operative mortality and morbidity risk models. Ann Thorac Surg 2003;75:1856-65. https://doi.org/10.1016/S0003-4975(03)00179-6

3. López Menéndez J, Polo López L, Silva Guisasola J, Centella Hernández T. Cirugía cardiovascular en España en el año 2016. Registro de intervenciones de la Sociedad Española de Cirugía Torácica-Cardiovascular. Cirugia Cardiovascular. 2017;24(6):38197. https://doi.org/10.1016/j.circv.2017.09.005

4. Dünser MW, Bouvet $\mathrm{O}$, Knotzer $\mathrm{H}$, Arulkumaran N, Hajjar LA, Ulmer $\mathrm{H}$, et al.Vasopressin in Cardiac Surgery: A Meta-analysis of Randomized Controlled Trials. Journal of Cardiothoracic and Vascular Anesthesia. 2018;32(5):2225-32. https://doi.org/10.1053/j. jvca.2018.04.006

5. Luis A. Muñoz, Paola A. Susunagab, Mario Gómez, Mario Villabón JJA. Inotrópicos en el postoperatorio de cirugía cardiovascular: estudio de corte transversal Luis. Rev Colomb Cardiol. 2014;21(4):258-63. https://doi.org/10.1016/j.rccar.2014.05.005

6. University Hospitals of Leicester. Management of Adults with Diabetes Undergoing Surgery and Elective Procedures - Guidelines [Internet]. Leicester: University Hospitals of Leicester; 2016 [citado 28 de junio de 2019]. Disponible en: https://secure.library.leicestershospitals.nhs.uk/PAGL/Shared\%20Documents/Diabetes\%20 Patients\%20Undergoing\%20Surgery\%20UHL\%20Guideline.pdf

7. Bangash MN, Kong M-L, Pearse RM. Use of inotropes and vasopressor agents in critically ill patients. Br J Pharmacol. abril de 2012;165(7):2015-33. https://doi.org/10.1111/j.14765381.2011.01588.x

8. Ahmed I, House CM, Nelson WB. Predictors of inotrope use in patients undergoing concomitant coronary artery bypass graft ( $C A B G$ ) and aortic valve replacement (AVR) surgeries at separation from cardiopulmonary bypass (CPB). J Cardiothorac Surg. 2009;4:1-6. https://doi.org/10.1186/1749-8090-4-24

9. Sherafati A, Mollazadeh R. Benefits and Harms of Beta Blockers in the Perioperative Period of Non-Cardiac Surgery: A Narrative Review. Archives of Neuroscience. 2017;4(4): e57718 https://doi. org/10.5812/archneurosci.57718
10. Godinho AS, Alves AS, Pereira AJ, Pereira TS. Review Article On-Pump versus Off-Pump Coronary-Artery Bypass Surgery : a Meta-Analysis. Arq Bras Cardiol. 2012;98(1):87-94. https://doi. org/10.1590/S0066-782X2012000100014

11. Pres Dres I,Gómez JM, Riva J, Kohn E, Bouchacourt JP, Hurtado J. Efecto de la circulación extracorpórea sobre la perfusión microvascular en pacientes sometidos a cirugía cardiaca. Anest Analg Reanim. 2011;24 (1):4-12.

12. Velissaris T, Tang AT, Murray M, Mehta RL, Wood PJ, Hett DA, et al. A prospective randomized study to evaluate stress response during beating-heart and conventional coronary revascularization. Ann Thorac Surg. 2004;78(2):506-12. https://doi. org/10.1016/S0003-4975(03)01360-2

13. Aguirre-Sánchez JS, Bucio-Reta E, Martínez- L, López-Pineda DM, Santos-Martínez LE, Florez-Figueroa F. Síndrome vasopléjico. Arch Cardiol Mex. 2011; 81(Supl.2):58-63. 14. Nearman $\mathrm{H}$, Klick JC, Eisenberg P, Pesa N. Perioperative complications of cardiac surgery and postoperative care. Critical Care Clinics. 2014;30(3):527-55. https://doi.org/10.1016/j.ccc.2014.03.008

15. POISE Study Group, Devereaux PJ, Yang H, Yusuf S, Guyatt G, Leslie K, et al. Effects of extended-release metoprolol succinate in patients undergoing non-cardiac surgery (POISE trial): a randomised controlled trial. Lancet. 2008;371(9627):1839-47. https://doi. org/10.1016/S0140-6736(08)60601-7

16. Wijeysundera DN, Duncan D, Nkonde-Price C, Virani SS, Washam JB, Fleischmann KE, et al. Perioperative Beta Blockade in Noncardiac Surgery: A Systematic Review for the 2014 ACC/ AHA Guideline on Perioperative Cardiovascular Evaluation and Management of Patients Undergoing Noncardiac Surgery. Circulation. 2014;130(24):2246-64. https://doi.org/10.1161/ CIR.0000000000000104

17. Blessberger $\mathrm{H}$, Kammler J, Domanovits $\mathrm{H}$, Schlager $\mathrm{O}$, Wildner B, Azar D, Schillinger M, Wiesbauer F SC. Perioperative betablockers for preventing surgery-related mortality and morbidity. Cochrane Database Syst Rev. 2018;(3):1-362. https://doi. org/10.1002/14651858.CD004476.pub3

18. Song S-W, Yi G, Lee S, Youn Y-N, Sul S-Y, Yoo K-J. Perioperative Indicators of Stress Response and Postoperative Inflammatory Complications in Patients Undergoing Off-Pump Coronary Artery Bypass Surgery. Circ J. 2008;72(12):1966-74. https://doi. org/10.1253/circj.CJ-08-0291

19. Abu-Assi E, López-López A, González-Salvado V, RedondoDiéguez A, Peña-Gil C, Bouzas-Cruz N, et al. The Risk of Cardiovascular Events After an Acute Coronary Event Remains High, Especially During the First Year, Despite Revascularization. Rev Esp Cardiol (Engl Ed). 2015;69(1):11-8. https://doi.org/10.1016/j. recesp.2015.06.016

20. Rau T, Heide R, Bergmann K, Wuttke H, Werner U, Feifel N, Eschenhagen T. Effect of the CYP2D6 genotype on metoprolol metabolism persists during long-term treatment. Pharmacogenetics 2002;12:465-72. https://doi.org/10.1097/00008571-20020800000007 